\title{
Genetic analysis and phosphoinositide 3-kinase/protein kinase B signaling pathway status in ovarian endometrioid borderline tumor samples
}

\author{
KOHEI NAKAMURA ${ }^{1}$, KENTARO NAKAYAMA ${ }^{1}$, MASAKO ISHIKAWA ${ }^{1}$, TOSHIKO MINAMOTO ${ }^{1}$, \\ TOMOKA ISHIBASHI ${ }^{1}$, EMI SATO ${ }^{1}$, KAORI SANUKI ${ }^{1}$, HITOMI YAMASHITA $^{1}$, RURIKO ONO ${ }^{1}$, KOUJI IIDA ${ }^{1}$, \\ RAZIA SULTANA $^{1}$, MOHAMMAD MAHMUD HOSSAIN ${ }^{1}$, NORIYOSHI ISHIKAWA ${ }^{2}$ and SATORU KYO ${ }^{1}$
}

Departments of ${ }^{1}$ Obstetrics and Gynecology, and ${ }^{2}$ Organ Pathology, Shimane University School of Medicine, Izumo, Shimane 693-8501, Japan

Received July 17, 2016; Accepted March 28, 2017

DOI: $10.3892 / \mathrm{ol} .2018 .8626$

\begin{abstract}
Ovarian endometrioid borderline tumors (EBTs) are extremely rare, and are thought to be precursors of endometrioid carcinoma, beginning as adenofibroma or endometriosis and progressing in a slow, stepwise manner. In endometrioid carcinomas, a high frequency of activating mutations in phosphatase and tensin homolog $(P T E N), \beta$-catenin or AT-rich interaction domain $1 \mathrm{~A}(A R I D I A)$ genes, and the activation of the phosphoinositide 3-kinase (PI3K)/protein kinase $\mathrm{B}$ (AKT) signaling pathway have been observed. However, the frequency of these alterations in EBTs and how they contribute to tumor progression remain unclear. To the best of our knowledge, this is the first study to assess the status of the PI3K/AKT signaling pathway in EBTs, in association with PTEN and ARIDIA mutations. PTEN mutations were observed in EBTs and also in the area of endometriosis without atypia. However, the PI3K/AKT signaling pathway was revealed to be activated only in EBTs. The observations of the present study suggest that the PTEN mutation represents an early event in EBT tumorigenesis, while additional genetic alterations may be necessary to activate the PI3K/AKT signaling pathway and induce the development of the invasive carcinoma.
\end{abstract}

\section{Introduction}

Ovarian endometrioid carcinomas account for only $10 \%$ of all ovarian carcinomas (1). The majority of ovarian endometrioid carcinomas are thought to be type I, low-grade tumors with

Correspondence to: Dr Kentaro Nakayama, Department of Obstetrics and Gynecology, Shimane University School of Medicine, Enyacho 89-1, Izumo, Shimane 693-8501, Japan

E-mail:kn88@med.shimane-u.ac.jp

Key words: endometrioid borderline tumor, phosphatase and tensin homolog, AT-rich interaction domain 1A, mutation, phosphoinositide 3-kinase/protein kinase B signaling pathway good prognosis (1). The precursor lesions of ovarian endometrioid carcinomas have been described as endometrioid borderline tumors (EBTs); however, EBTs are extremely rare and constitute only $0.2 \%$ of all epithelial ovarian tumors. EBTs exhibit two major growth patterns: Adenofibromatous and intracystic (1). The adenofibromatous type arises from benign ovarian adenofibroma, whereas the intracystic types arise from the transformation of endometriosis, as revealed by their frequent coexistence in surgical specimens (2). The process underlying the molecular pathogenesis of EBT remains unclear, whereas a number of molecular alterations have been described in ovarian endometrioid carcinomas, including mutations in $\beta$-catenin (16-38\%) (3-7), phosphatase and tensin homolog $(P T E N ; 14-21 \%)(8,9)$, AT-rich interaction domain 1A (ARIDIA; 30\%) (10), phosphoinositide-3-kinase catalytic $\alpha$ polypeptide (PIK3CA; 0\%) (11) and tumor protein p53 (TP53; 60\%) $(6,12)$.

Mutations in the tumor-suppressor gene PTEN have been reported in a relatively high percentage of endometrial and ovarian cancer cases, particularly in the endometrioid subtype $(9,13,14)$. The majority of these mutations were observed in stage I tumors, indicating that PTEN inactivation represents an early event during ovarian tumorigenesis (9). However, PTEN mutations in EBTs have not previously been described, possibly due to their rarity. PTEN, located at chromosome 10q23, is a tumor-suppressor gene encoding a lipid phosphatase that dephosphorylates phosphatidylinositol 3,4,5-trisphosphate [PI(3,4,5)P3], performing opposing actions to phosphoinositide 3-kinase (PI3K) $(15,16)$. PI(3,4,5) $\mathrm{P} 3$ is converted to $\mathrm{PI}(3,4) \mathrm{P} 2$, which activates the proto-oncogene protein kinase B (PKB; also known as AKT). When phosphorylated, AKT becomes activated and antagonizes apoptotic pathways via the activation of mechanistic target of rapamycin or the inactivation of the members of the forkhead family, whereas the dephosphorylation of PI(3,4,5)P3 by PTEN induces the activation of the apoptotic pathway (17-19). Therefore, the fundamental role of PTEN is the inhibition of the PI3K/AKT signaling pathway. PTEN mutations may disrupt this inhibitory role, thereby inducing the antiapoptotic pathway. 
BAF250a, the protein encoded by ARIDIA $(20,21)$, is one of the accessory subunits of the SWItch/Sucrose Non-Fermentable complex (SWI/SNF). SWI/SNF is a chromatin-remodeling complex that is found in all eukaryotes and is responsible for regulating gene expression during numerous cellular processes, including differentiation, development, DNA repair, proliferation, and tumor suppression (22). Using adenosine triphosphate, SWI/SNF mobilizes nucleosomes, thereby modulating the accessibility of promoters for transcriptional activation or repression. Rearrangement of ARIDIA has been demonstrated in a breast cancer cell line, while a lung cancer cell line has been shown to exhibit $A R I D I A$ deletion, indicating that $A R I D I A$ is a tumor-suppressor gene (22). The nature and pattern of ARIDIA mutations in ovarian endometrioid carcinoma also provide evidence of its role as a tumor suppressor (10). In a previous study, ARIDIA mutations were revealed to frequently co-occur with $P I K 3 C A$ gene mutations and PI3K/AKT signaling pathway activation, as demonstrated by the increased AKT1 activation in endometrial cancer (23). However, to the best of our knowledge, the roles of ARIDIA mutation in EBTs have not previously been described. Therefore, the majority of these genetic alterations, which are prevalent in ovarian endometrioid carcinomas, induce the activation of the PI3K/AKT signaling pathway, and may serve major roles in carcinogenesis as well as disease progression.

The aim of the present study was to investigate the PI3K/AKT signaling pathway status in EBTs and the role of $P T E N$ and ARIDIA mutations. The present study examined the differences in PTEN and ARIDIA mutations between benign and borderline patient tissue samples in order to gain insight into the molecular pathogenesis involved in the initiation of EBT.

\section{Materials and methods}

Tissue samples. Formalin-fixed, paraffin-embedded (FFPE) tissue samples isolated from two cases of EBT of the ovaries were analyzed in the present study. Samples were obtained from the Department of Obstetrics and Gynecology at the Shimane University Hospital (Izumo, Japan) between March 2013 and December 2015. The patients were a 61-year-old female (case 1) and a 43-year-old female (case 2) who had presented with abdominal distension as the chief complaint. The patients had undergone laparoscopic bilateral salpingo-oophorectomy, and had no significant previous medical or family history of disease.

The resected specimens were sectioned (section thickness, $3 \mu \mathrm{m}$ ), stained with hematoxylin and eosin and reviewed by a pathologist. Subsequently, the tissue specimens were immunohistochemically stained with the following antibodies overnight at $4^{\circ} \mathrm{C}$ : Monoclonal anti-PTEN antibody (cat. no. ABM-2052; 1:100; Cascade BioScience, Inc., Winchester, MA, USA), anti-ARID1A (cat. no. sc-32761; 1:50; Santa Cruz Biotechnology, Inc., Dallas, TX, USA), anti-p53 (cat. no. 760-2542; 1:200; Roche Diagnostics, Indianapolis, IN, USA), anti- $\beta$-catenin (cat. no. ab32572; 1:1,000; Abcam, Cambridge, UK) and anti-phosphorylated (p)-AKT (cat. no. 4060; Ser473; 1:50; Cell Signaling Technology, Inc., Danvers, MA, USA) antibodies. The following day, slides were washed three times with PBS prior to the detection of antigens using the two-step DAKO EnVision+ Peroxidase System (DAKO; Agilent Technologies, Inc., Santa Clara, CA, USA), according to the manufacturer's protocol, at room temperature for $30 \mathrm{~min}$. After rinsing with PBS, the sections were incubated at room temperature for $5 \mathrm{~min}$ in $0.05 \%$ diaminobenzidine in PBS with $0.03 \% \mathrm{H}_{2} \mathrm{O}_{2}$.

Acquisition of tissue specimens was approved by the Ethics Committee of Shimane University School of Medicine (approval no. 2004-0381). Written informed consent was provided by all participants prior to enrollment in the present study. This study was conducted in accordance with the Declaration of Helsinki and Title 45, U.S. Code of Federal Regulations, Part 46, Protection of Human Subjects, effective December 13, 2001.

Scoring of immunohistochemical staining. All samples were evaluated by two pathologists in Shimane University Hospital, who were blinded to the present study. The samples were scored negative for PTEN when complete loss of expression in the tumor sample was observed, with stromal cells and normal fallopian tube epithelial cells used as the positive controls. PTEN staining was considered positive when strong, weak or heterogeneous staining was observed.

Absence of nuclear staining of ARID1A is referred to as a 'clonal loss' pattern and corresponds to mutations in ARID1A (10); therefore, such tissues were scored as 'loss of expression'. Surrounding stromal cells and normal fallopian epithelial cells served as positive controls.

p53 was scored 'wild-type-like' when $<50 \%$ of the tumor cells demonstrated positive nuclear staining. p53 was scored 'mutant-like' if $>50 \%$ of the tumor cells exhibited strong positive nuclear staining or when discrete geographical patterns exhibited $>50 \%$ tumor cell positivity.

DNA extraction. Borderline regions of both cases contained sufficient tumor tissue to extract DNA and perform sequence analysis. Benign epithelial regions were not observed in case 1. Tissue sections were placed on membrane slides and counterstained with hematoxylin for $1 \mathrm{~min}$ at room temperature. Selected tumor tissues were dissected, and after $48 \mathrm{~h}$ of digestion with Proteinase K solution (cat. no. 25530-049; Invitrogen; Thermo Fisher Scientific, Inc., Waltham, MA, USA) at $56^{\circ} \mathrm{C}$, DNA was extracted from the dissected samples using a QIAamp DNA Micro kit (Qiagen Inc., Valencia, CA, USA), according to the manufacturer's protocol.

Sequence analysis. PCR amplification was performed for all 9 exons of PTEN using primers (Table I) and genomic DNA from dissected FFPE tissue. PCR amplification was performed in $20 \mu 1$ reaction volumes that contained $75 \mathrm{mM}$ Tris- $\mathrm{HCl}, 1.5 \mathrm{mM} \mathrm{MgCl} 2,50 \mathrm{mM} \mathrm{KCl}, 20 \mathrm{mM}(\mathrm{NH} 4)_{2} \mathrm{SO}_{4}$, $0.2 \mathrm{mM}$ of each primer, and $1 \mathrm{U}$ Taq DNA polymerase (Takara Bio, Inc., Otsu, Japan). The PCR experiments were conducted under the following conditions: An initial 5 min denaturation at $95^{\circ} \mathrm{C} ; 35$ cycles of $1 \mathrm{~min}$ each at 94,57 , and $72^{\circ} \mathrm{C}$; and a single final extension step for $10 \mathrm{~min}$ at $72^{\circ} \mathrm{C}$. Amplified PCR products were sequenced at Beckman Coulter, Inc. (Brea, CA, USA) and analyzed using the Mutation Surveyor DNA Variant 
Table I. Primers used for PCR amplification of the PTEN gene.

\begin{tabular}{lll} 
Exon & \multicolumn{1}{c}{ Forward primer 5'-3' } & \multicolumn{1}{c}{ Reverse primer 5'-3' } \\
\hline 1 & TTCCATCCTGCAGAAGAAGC & CAGCCGCAGAAATGGATAC \\
2 & ACTCCAGCTATAGTGGGGAAA & TTTTCTGGGCTTAGAAATCTTT \\
3 & TGATTACTACTCTAAACCCATAGAAGG & TTGTTTTAGAAGATATTTGCAAGC \\
4 & AAAGATTCAGGCAATGTTTGTT & TCTCACTCGATAATCTGGATGAC \\
5 & TCCAGTGTTTCTTTTAAATACCTGTT & GATCCAGGAAGAGGAAAGGAA \\
6 & ATATATGTTCTTAAATGGCTACGA & ACATGGAAGGATGAGAATTTC \\
7 & TCATTAAAATCGTTTTTGACAGTTT & TCTGTCCTTATTTTGGATATTTCTC \\
8 & TGTTTAACATAGGTGACAGATTTTCTT & ACAAGTCAACAACCCCCACA \\
9 & TGTTCATCTGCAAAATGGAATAA & CACAATGTCCTATTGCCATTAAA
\end{tabular}

Analysis Software (version 4.0.6; SoftGenetics LLC, State College, PA, USA).

\section{Results}

Clinical findings. The tissue samples were obtained from a 61-year-old female (case 1) and a 43-year-old female (case 2) with abdominal distension as the chief complaint. The patients underwent laparoscopic bilateral salpingo-oophorectomy, and had no significant previous medical or family history of disease. The serum cancer antigen 125 expression levels were within normal limits $(<35.0 \mathrm{U} / \mathrm{ml})$, and the results of the general examination were also normal. Following the initial surgical procedure, the patients underwent total hysterectomy and omentectomy due to the EBT diagnosis. Follow-up data was available in case 1 for 5 years and in case 2 for 2 years. The two patients were alive and well at the last follow-up.

\section{Pathological findings}

Case 1. Gross observations of the tumor of the right ovary revealed that it had a maximum diameter of $7.5 \mathrm{~cm}$. Microscopically, the tumor was categorized as having an endometrioid adenofibromatous growth pattern. Numerous glands with intervening fibrous stroma were observed and these glands demonstrated varying degrees of endometrial hyperplasia, ranging from simple hyperplasia to a more marked proliferation, similar to that observed in complex atypical hyperplasia (Fig. 1). No invasion of the ovarian stroma was identified.

Case 2.Gross observations of the tumor in the left ovary revealed that its maximum diameter was $4.5 \mathrm{~cm}$. Microscopically, the tumor was categorized as having an intracystic growth pattern. The tumor exhibited a papillary growth pattern protruding into a cystic space (Fig. 2A). Cells with moderate cytological atypia were observed. Atypical endometrial glands were observed in the endometrial background (Fig. 2B). No invasion of the ovarian stroma was identified.

Immunohistochemical findings. Loss of PTEN protein expression was revealed in the nuclei of neoplastic cells in both EBTs. In case 2, loss of PTEN expression was identified not only in the area of endometriosis with atypia, but also endometriosis without atypia (Fig. 3).

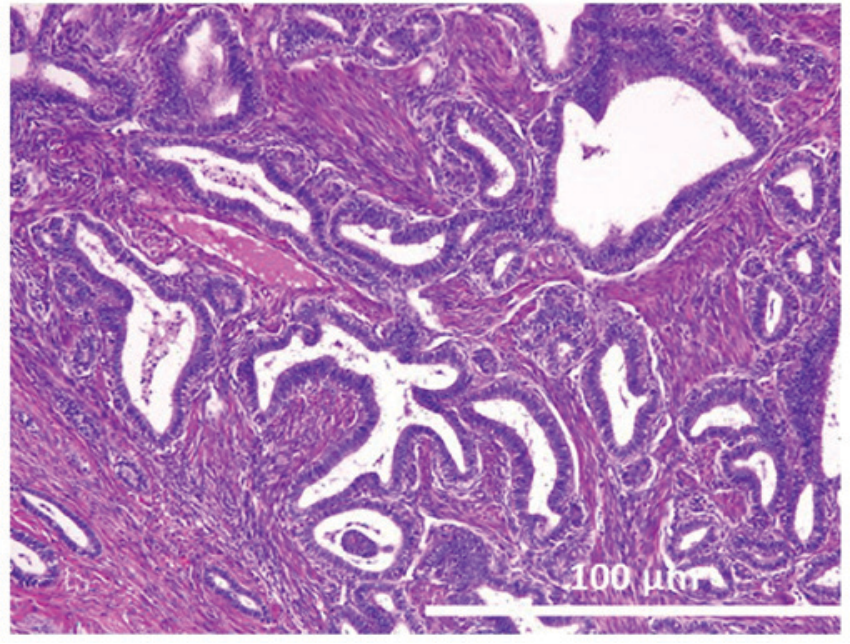

Figure 1. Case 1: Hematoxylin and eosin staining of endometrioid borderline tumor (adenofibromatous type). Mild cytological atypia is present in the endometrioid glands (magnification, x400).

Loss of ARID1A protein expression was observed in the nuclei of neoplastic cells, whereas strong expression was maintained in stromal and normal fallopian tube epithelial cells of the same section in case 1 (Fig. 4). In case 2, ARID1A expression was revealed to be present, and was most likely wild-type protein. Wild-type p53 and $\beta$-catenin expression patterns were observed in both tissue samples.

Furthermore, the expression level of p-AKT in these two cases was assessed. The tumor cells in case 1 were negative for p-Akt expression (Fig. 5A); however, in case 2, p-AKT expression was not identified in the area of endometriosis without atypia, but was in the endometriosis with atypia (Fig. 5B and C).

PTEN mutational analysis. PCR amplification and sequence analysis were performed for the borderline regions in case 1 tissue samples, and in the benign and borderline regions in the case 2 tissue samples. The borderline regions of both samples were revealed to harbor a c.483insG mutation in exon 8 of the PTEN gene (Fig. 6). Regarding the regions of endometriosis without atypia, the same mutations were detected. c.483insG represents a frameshift mutation that 

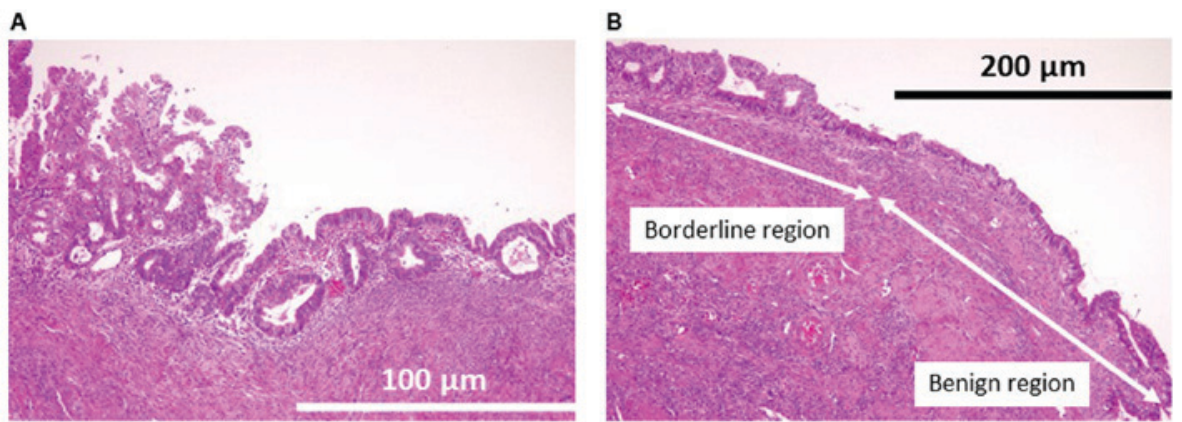

Figure 2. Case 2: Hematoxylin and eosin staining of endometrioid borderline tumor (intracystic type). (A) Endometriosis with atypia demonstrated papillary growth pattern (magnification, x400). (B) Endometriosis with/without atypia (magnification, x200).

A

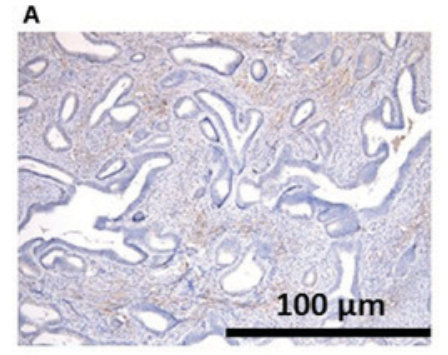

C

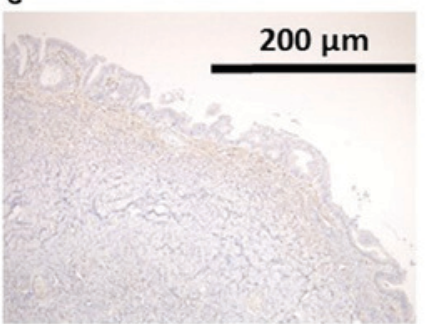

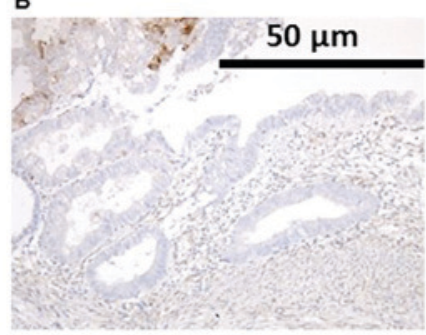

D

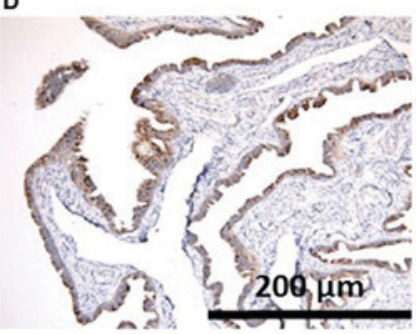

Figure 3. Phosphatase and tensin homolog immunostaining. (A) Case 1, tumor (negative) (magnification, $\mathrm{x} 400$ ). (B) Case 2, benign regions (negative) (magnification, x800). (C) Case 2, borderline tumor (negative) (magnification, $\mathrm{x} 200$ ). (D) Fallopian tube epithelial cells (positive control) (magnification, $\mathrm{x} 200)$.

A

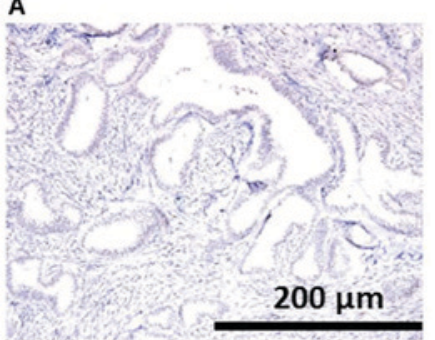

B

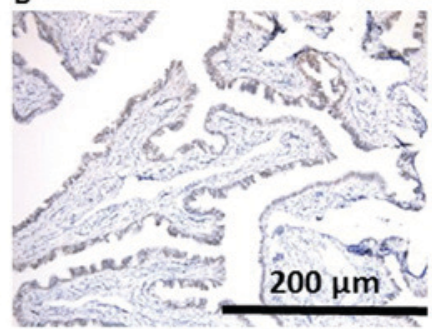

Figure 4. AT-rich interaction domain 1A immunostaining. (A) Case 1 tissue sample (negative) (magnification, x200). (B) Fallopian tube epithelial cells (positive control) (magnification, $\mathrm{x} 200$ ).

results in the formation of a stop codon (p.ASP485X) for PTEN protein translation.

\section{Discussion}

There are few previous studies investigating EBTs due to their rarity; to the best of our knowledge, only two series and
A

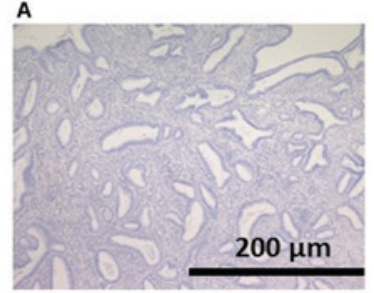

C

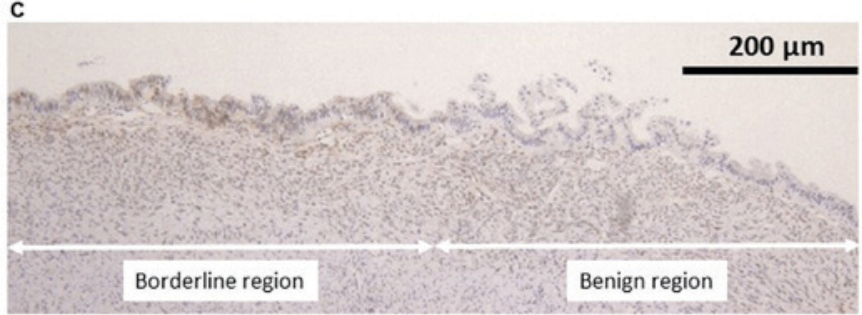

Figure 5. Phosphorylated protein kinase B immunostaining. (A) Case 1 tissue sample (negative) (magnification, x200). (B) Case 2 tissue sample including benign and borderline regions (benign regions: negative, borderline regions: positive) (magnification, x200). (C) Borderline regions in case 2 tissue sample (positive) (magnification, $\mathrm{x} 400$ ).

A

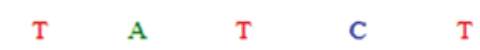

c.483insG

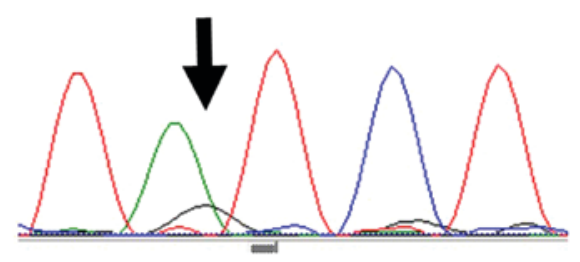

$\begin{array}{llllll}\text { B } & \text { T } & \text { A } & \text { T } & \text { C } & \text { T }\end{array}$

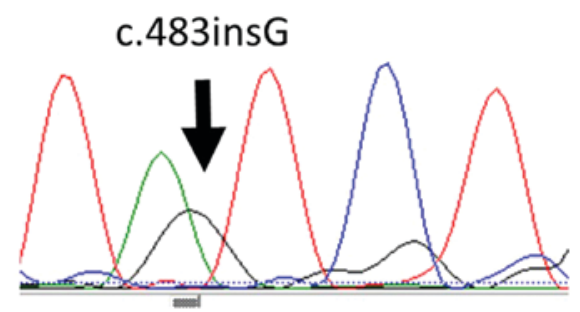

Figure 6. DNA sequencing electropherograms showing the sequence of exon 8 of the phosphatase and tensin homolog gene sequenced from (A) case 1 and (B) case 2 tissue samples. The arrow indicates the insertion mutation at c.483insG. 
Table II. Genetic analyses of distinct tumor regions.

\begin{tabular}{|c|c|c|c|c|c|c|}
\hline Case no. & Tissue region & PTEN & ARID1A & $\beta$-catenin & p53 & $\mathrm{p}-\mathrm{AKT}$ \\
\hline Case 1 & Borderline & Mutant & Mutant & WT & WT & Negative \\
\hline \multirow[t]{2}{*}{ Case 2} & Benign (endometriosis without atypia) & Mutant & WT & WT & WT & Negative \\
\hline & Borderline (endometriosis with atypia) & Mutant & WT & WT & WT & Positive \\
\hline
\end{tabular}

PTEN, phosphatase and tensin homolog; ARID1A, AT-rich interaction domain 1A; p-AKT, phosphorylated protein kinase B; WT, wild-type,

one case study have been previously reported (24-26). EBTs were observed in the right and left ovaries in $2-5 \%$ of cases at the time of diagnosis in these studies, and the predominant growth pattern was adenofibromatous in both case series, as well as in case 1 of the present study. Among all of these studies, only case 2 of the present study was demonstrated to be associated with endometriosis. Additionally, glandular and papillary proliferations with a high grade of complexity and mild atypia in the lining epithelial cells were identified in the present study. In all of the cases mentioned, no squamous differentiation was observed and there was no stromal invasion; the growth was limited to only one ovary with the capsule intact. In the long-term follow-up, both of the case series of EBTs demonstrated no signs of recurrence or metastasis, whereas $20 \%$ of patients with well-differentiated endometrioid carcinoma were revealed to have recurrence (24). It has been observed that the endometrioid neoplasms of the ovary are usually carcinomas, whereas borderline tumors are extremely rare, and are thought to arise from adenofibroma or endometriosis (1).

Ovarian endometrioid carcinomas are genetically stable and have been described to arise from EBT (1). They are associated with PTEN, ARIDIA, PIK3CA and TP53 mutations (1). $P T E N$ mutations may represent an early event in the pathogenesis of endometrioidcarcinoma (27). However, the frequency of PTEN mutation in EBTs has not been described previously. These prevalent genetic alterations observed in ovarian endometrioid carcinoma are known to induce the activation of the PI3K/AKT signaling pathway (6). However, as EBTs are extremely rare, the status of genetic mutations in these tumors is yet to be reported.

The present study analyzed the status of genetic mutations in EBTs and their association with the activation of the PI3K/AKT signaling pathway for the first time. In case 1, which was associated with adenofibroma, PTEN and ARIDIA mutations were observed. However, in case 2, which was associated with endometriosis, ARIDIA mutation was not observed, whereas PTEN mutation was observed in EBT as well as in the area of endometriosis without atypia, although the PI3K/AKT signaling pathway was activated only in the EBT area. The results of genetic alteration analyses are provided in Table II. The observations suggest that the PTEN mutation represents an early event in EBT tumorigenesis, whereas the additional genetic alterations may be necessary in order for the PI3K/AKT signaling pathway to be activated and induce the development of EBT to invasive carcinoma. Additional studies will be required to confirm the findings and implications of the present study.

\section{Acknowledgements}

Not applicable.

\section{Funding}

No funding was received.

\section{Availability of data and materials}

The datasets used and/or analyzed during the current study are available from the corresponding author on reasonable request.

\section{Authors' contributions}

KoN drafted the manuscript and carried out the molecular genetic studies. MI, TM, TI, ES, KS and HY carried out the molecular genetic studies and participated in the sequence alignment. RO, KI, RS, MMH and NI carried out the staining. KeN participated in the design of the study. SK conceived the study, and participated in its design and coordination and helped to draft the manuscript. All authors read and approved the final manuscript.

\section{Ethics approval and consent to participate}

The study protocol was approved by the Ethics Committee of Shimane University School of Medicine (Izumo, Japan) (approval no. 960). All patients provided informed written consent. The research was conducted in accordance with the Declaration of Helsinki and Title 45, US Code of Federal Regulations, Part 46, Protection of Human Subjects, effective December 13, 2001.

\section{Consent for publication}

All patients provided written informed consent for the publication of data in this study.

\section{Competing interests}

The authors declare that they have no competing interests.

\section{References}

1. Chen S, Leitao MM, Tornos C and Soslow RA: Invasion patterns in stage I endometrioid and mucinous ovarian carcinomas: A clinicopathologic analysis emphasizing favorable outcomes in carcinomas without destructive stromal invasion and the occasional malignant course of carcinomas with limited destructive stromal invasion. Mod Pathol 18: 903-911, 2005. 
2. Prowse AH, Manek S, Varma R, Liu J, Godwin AK, Maher ER, Tomlinson IP and Kennedy SH: Molecular genetic evidence that endometriosis is a precursor of ovarian cancer. Int J Cancer 119: $556-562,2006$

3. Moreno-Bueno G, Gamallo C, Pérez-Gallego L, de Mora JC, Suárez A and Palacios J: Beta-catenin expression pattern, beta-catenin gene mutations, and microsatellite instability in endometrioid ovarian carcinomas and synchronous endometrial carcinomas. Diagn Mol Pathol 10: 116-122, 2001.

4. Sagae S, Kobayashi K, Nishioka Y, Sugimura M, Ishioka S, Nagata M, Terasawa K, Tokino T and Kudo R: Mutational analysis of beta-catenin gene in Japanese ovarian carcinomas: Frequent mutations in endometrioid carcinomas. Jpn J Cancer Res 90: 510-515, 1999.

5. Wright K, Wilson P, Morland S, Campbell I, Walsh M, Hurst T, Ward B, Cummings M and Chenevix-Trench G: Beta-catenin mutation and expression analysis in ovarian cancer: Exon 3 mutations and nuclear translocation in $16 \%$ of endometrioid tumours. Int J Cancer 82: 625-629, 1999.

6. Wu R, Hendrix-Lucas N, Kuick R, Zhai Y, Schwartz DR, Akyol A, Hanash S, Misek DE, Katabuchi H, Williams BO, et al: Mouse model of human ovarian endometrioid adenocarcinoma based on somatic defects in the Wnt/beta-catenin and PI3K/Pten signaling pathways. Cancer Cell 11: 321-333, 2007.

7. Wu R, Zhai Y, Fearon ER and Cho KR: Diverse mechanisms of beta-catenin deregulation in ovarian endometrioid adenocarcinomas. Cancer Res 61: 8247-8255, 2001.

8. Catasus L, Bussaglia E, Rodrguez I, Gallardo A, Pons C, Irving JA and Prat J: Molecular genetic alterations in endometrioid carcinomas of the ovary: Similar frequency of beta-catenin abnormalities but lower rate of microsatellite instability and PTEN alterations than in uterine endometrioid carcinomas. Hum Pathol 35: 1360-1368, 2004.

9. Obata K, Morland SJ, Watson RH, Hitchcock A, Chenevix-Trench G, Thomas EJ and Campbell IG: Frequent PTEN/MMAC mutations in endometrioid but not serous or mucinous epithelial ovarian tumors. Cancer Res 58: 2095-2097, 1998.

10. Wiegand KC, Shah SP, Al-Agha OM, Zhao Y, Tse K, Zeng T, Senz J, McConechy MK, Anglesio MS, Kalloger SE, et al: ARID1A mutations in endometriosis-associated ovarian carcinomas. N Engl J Med 363: 1532-1543, 2010.

11. Campbell IG, Russell SE, Choong DY, Montgomery KG, Ciavarella ML, Hooi CS, Cristiano BE, Pearson RB and Phillips WA: Mutation of the PIK3CA gene in ovarian and breast cancer. Cancer Res 64: 7678-7681, 2004.

12. Schwartz DR, Kardia SL, Shedden KA, Kuick R, Michailidis G, Taylor JM, Misek DE, Wu R, Zhai Y, Darrah DM, et al: Gene expression in ovarian cancer reflects both morphology and biological behavior, distinguishing clear cell from other poor-prognosis ovarian carcinomas. Cancer Res 62: 4722-4729, 2002.

13. Tashiro H, Blazes MS, Wu R, Cho KR, Bose S, Wang SI, Li J, Parsons R and Ellenson LH: Mutations in PTEN are frequent in endometrial carcinoma but rare in other common gynecological malignancies. Cancer Res 57: 3935-3940, 1997.

14. Risinger JI, Hayes AK, Berchuck A and Barrett JC: PTEN/MMAC1 mutations in endometrial cancers. Cancer Res 57: 4736-4738, 1997.
15. MaehamaT and Dixon JE: The tumor suppressor,PTEN/MMAC1, dephosphorylates the lipid second messenger, phosphatidylinositol 3,4,5-trisphosphate. J Biol Chem 273: 375-378, 1998.

16. Myers MP, Pass I, Batty IH, Van der Kaay J, Stolarov JP, Hemmings BA, Wigler MH, Downes CP and Tonks NK: The lipid phosphatase activity of PTEN is critical for its tumor supressor function. Proc Natl Acad Sci USA 95: 13513-13518, 1998.

17. Franke TF, Kaplan DR and Cantley LC: PI3K: Downstream AKTion blocks apoptosis. Cell 88: 435-437, 1997.

18. Downward J: Ras signalling and apoptosis. Curr Opin Genet Dev 8: 49-54, 1998.

19. Brunet A, Bonni A, Zigmond MJ, Lin MZ, Juo P, Hu LS, Anderson MJ, Arden KC, Blenis J and Greenberg ME: Akt promotes cell survival by phosphorylating and inhibiting a Forkhead transcription factor. Cell 96: 857-868, 1999.

20. Sif S, Saurin AJ, Imbalzano AN and Kingston RE: Purification and characterization of $\mathrm{mSin} 3 \mathrm{~A}$-containing Brg 1 and $\mathrm{hBrm}$ chromatin remodeling complexes. Genes Dev 15: 603-618, 2001.

21. Wang W, Xue Y, Zhou S, Kuo A, Cairns BR and Crabtree GR: Diversity and specialization of mammalian SWI/SNF complexes. Genes Dev 10: 2117-2130, 1996.

22. Reisman D, Glaros S and Thompson EA: The SWI/SNF complex and cancer. Oncogene 28: 1653-1668, 2009.

23. Liang H, Cheung LW, Li J, Ju Z, Yu S, Stemke-Hale K, Dogruluk T, Lu Y, Liu X, Gu C, et al: Whole-exome sequencing combined with functional genomics reveals novel candidate driver cancer genes in endometrial cancer. Genome Res 22: 2120-2129, 2012 .

24. Roth LM, Emerson RE and Ulbright TM: Ovarian endometrioid tumors of low malignant potential: A clinicopathologic study of 30 cases with comparison to well-differentiated endometrioid adenocarcinoma. Am J Surg Pathol 27: 1253-1259, 2003.

25. Bell KA and Kurman RJ: A clinicopathologic analysis of atypical proliferative (borderline) tumors and well-differentiated endometrioid adenocarcinomas of the ovary. Am J Surg Pathol 24: $1465-1479,2000$

26. Jetley S, Khetrapal S, Ahmad A and Jairajpuri ZS: Atypical proliferative endometrioid tumor of ovary: Report of a rare case. J Postgrad Med 62: 129-132, 2016.

27. Levine RL, Cargile CB, Blazes MS, van Rees B, Kurman RJ and Ellenson LH: PTEN mutations and microsatellite instability in complex atypical hyperplasia, a precursor lesion to uterine endometrioid carcinoma. Cancer Res 58: 3254-3258, 1998.

This work is licensed under a Creative Common Attribution-NonCommercial-NoDerivatives 4.0 International (CC BY-NC-ND 4.0) License. 